\section{PTU-111 LIP-RMDVA COMPOSED OF LIPOSOMAL CDNA ENCODING RECOMBINANT MULTIMODULAR PROTEINS RMDVA COMPRISING DISINTEGRIN/ CYSTEINE RICH DISULFIDE BOND 2RGD, C-TERMINAL DOMAIN, METALLOPROTEASE-DOMAIN, AND DIMERIC DISINTEGRIN/MLD-VGD DOMAIN ERADICATED CRC}

doi:10.1136/gut.2011.239301.239

J Giannios* Translational Cancer Medicine, Erasinio Oncology Hospital, Athens, Greece

Introduction We investigate if venom proteome of vipera ammodytes can induce apoptosis in CRC.

Methods We developed a large scale expression system with CRC cell line which we modified genetically by cloning. We expressed cDNA encoding multimodular disintegrin (rMDVA) comprising 3 domains from the venom of vipera ammodytes. The cDNA of rMDVA was cloned into the plasmid vector pcDNAIII, which was transferred into CRC. The multimodular peptide rMDVA was encapsulated in the hydrophilic phase of liposomes termed as LIP-rMDVA. The in vivo studies with human metastatic CRC were performed in an orthotopic xenograft-model.

Results The venom proteome contained 140 proteins. We isolated the multimodular disintegrin displaying cysteine rich disulfide bond 2RGD and C-terminal motif (ammodystatin), the dimeric MLD/VGD motif (VADD), and metalloproteasedomain (ammodylysin). Ammodystatin with the 2 (ARGGLY-ASP) RGD motifs interacts with integrins avb5, a5b1 inhibiting invasion and angiogenesis/vasculogenesis. It also blocked the bFGF-induced avb3 integrin and avb5 downregulating VEGF, and Ras. It also disrupts the actin cytoskeleton inducing G1 DNA replicative phase arrest, and blocks endothelial cell (EC) secretion of MMP-2, MMP-9 and urokinase-type plasminogen activator with its receptor inhibiting proteolysis and dissolution of ECM blocking invasion. Ammodystatin and ammodylysin lead to type I PCD/apoptosis of tumour cells in masses up to $2 \mathrm{~mm}$ and EC. Beyond that size, ammodystatin inhibits the development of the required vascular network according to HUVEC, MVD and IHC. VADD causes burring and blocked adhesion of a $4 b 1$ integrin to VCAM1. LDH, BrdU and MTT exhibited inhibition of tumour and EC proliferation. TEM exhibited apoptotic signs of irreversible D2 stage in tumour and ECs. 
Conclusion Novel liposomal formulation LIP-rMDVA is an effective angiostatic and cytostatic agent against metastatic CRC inducing apoptosis with minimum systemic toxicity.

Competing interests None.

Keywords venom proteome, liposomal cDNA, angiogenesis, cytoskeleton, apoptosis, CRC. 